\title{
Efficacy and safety of pemetrexed on recurrent primary central nervous system lymphomas in China: a prospective study
}

This article was published in the following Dove Press journal:

OncoTargets and Therapy

17 May 2017

Number of times this article has been viewed

\section{Yi Sun ${ }^{1-3, *}$ \\ Yong Wang ${ }^{2, *}$ \\ Shaolong $\mathrm{Han}^{3}$ \\ Bo Xing ${ }^{3}$ \\ Hong $\mathrm{Li}^{4}$ \\ Yufang Zhu ${ }^{2}$ \\ Shizhen Zhou ${ }^{2}$ \\ Xiuhua Wang ${ }^{2}$ \\ Jun $X u^{2}$ \\ Rongjie $\mathrm{TaO}^{2}$}

'School of Medicine and Life Sciences, University of Jinan, Shandong

Academy of Medical Sciences,

${ }^{2}$ Department of Neurosurgery,

Shandong Cancer Hospital affiliated

to Shandong University, ${ }^{3}$ Department

of Neurosurgery, The Sixth People's

Hospital of Jinan, ${ }^{4}$ Department of

Ophthalmology and Otolaryngology,

The Sixth People's Hospital of

Jinan, Jinan City, Shandong Province,

People's Republic of China

*These authors contributed equally to this work
Correspondence: Rongjie Tao; Jun Xu Department of Neurosurgery, Shandong Cancer Hospital affiliated to Shandong University, Shandong Academy of Medical Sciences, Jinan 250I17, People's

Republic of China

Email II5438779@qq.com
Background: Pemetrexed, a new and novel agent for primary central nervous system lymphomas (PCNSLs), has shown to be efficient as a savage therapy for recurrent PCNSLs. However, more studies are needed. A prospective study was performed on 17 recurrent PCNSL patients with pemetrexed at Shandong Tumor Hospital in China to assess the efficacy and safety of pemetrexed for recurrent PCNSL patients.

Materials and methods: The medical records and imaging data on all the cases of recurrent PCNSL patients with pemetrexed in our study were collected during August 2012 and April 2015. Folic acid, $B_{12}$, and dexamethasone were used to induce toxicities related to pemetrexed. Patients were treated with pemetrexed at a dose of $900 \mathrm{mg} / \mathrm{m}^{2}$ intravenously every 3 weeks, and one cycle consists of 6 weeks.

Results: A total of 17 cases of recurrent PCNSL patients were enrolled in our study, including 10 males and 7 females with a median age of 66.2 years (ranging from 35 to 81). After the treatment, five cases had complete remission, with partial remission in five cases, stable disease in four cases, and progressive disease in three cases. Consequently, the overall response rate was $58.8 \%$, and the disease control rate was $82.4 \%$. The median overall survival was 7.8 months (95\% confidence interval: 5.9-9.6 months) in the study of recurrent PCNSL patients.

Conclusion: This study has been the first clinical trial that applied pemetrexed to treat recurrent PCNSL patients in China, and results indicated that chemotherapy using large pemetrexed may become an effective treatment for PCNSL recurrence with modest toxicity.

Keywords: primary central nervous system lymphomas, efficacy, safety, recurrence

\section{Introduction}

Primary central nervous system lymphoma (PCNSL), an aggressive extranodal non-Hodgkin's lymphoma, is confined within the central nervous system which includes brain, meninges, spinal cord, and eyeball, causing various neurological symptoms and signs. Although being a rare disease, the incidence of PCNSL has represented a rising trend in recent years. Due to the increasing incidence of PCNSL, a percentage rate ranging from $1 \%$ to $4 \%$ among the nonimmunodeficient patients with malignant intracranial tumors was related to PCNSL. In addition, the most common histologic type is the diffuse large B-cell lymphoma among the PCNSL patients under an average age of 60 years old. ${ }^{1,2}$

In fact, nearly $50 \%$ of patients will relapse within 24 months after diagnosis. Moreover, in situ recurrence was commonly observed among the PCNSL patients, followed by other intracranial metastasis, even spinal cord and eyeball metastasis. Despite numerous approaches being tried using radiation therapy (RT) or chemotherapy 
with variable degrees of activity, the best therapeutic strategy has still been a matter of debate. Unfortunately, the previous aggressive therapies of relapsed PCNSL patients often had poor prognosis responses. ${ }^{3-7}$ Pemetrexed, a new and novel chemotherapy agent for PCNSL, has shown to be efficient as a savage therapy for recurrent PCNSL. ${ }^{8,9}$ However, substantially more experience should be gained in evaluating the efficacy of pemetrexed for recurrent PCNSL patients. In this study, a prospective investigation was performed on 17 recurrent PCNSL patients with pemetrexed at Shandong Cancer Hospital in China to assess the efficacy of pemetrexed in these patients and explore a more effective second-line therapy for PCNSLs with few side effects.

\section{Materials and methods Study cases}

The study and clinical trial were approved by the ethical review board of Shandong Cancer Hospital. After written informed consent was obtained from each patient, the medical records and imaging data on all the cases of recurrent PCNSL patients with pemetrexed were collected during August 2012 and April 2015 at Shandong Cancer Hospital in Jinan, China. The diagnostic criteria of recurrent PCNSL in this study were defined that after the treatment, the PCNSL patients proven initially with biopsy had clinical symptoms and imaging features of PCNSL again. According to the imaging examination, the recurrent PCNSL patients in our study were all diagnosed through magnetic resonance imaging (MRI) and magnetic resonance spectroscopy (MRS). All the patients were initially biopsy proven and were negative for immune system diseases and HIV. Moreover, after the diagnosis confirmation, chemotherapy was selected as the first choice for the treatment, but if the disease progression cannot be controlled by chemotherapy, radiotherapy would be used to all the recurrent PCNSL patients.

\section{Inclusion and exclusion criteria}

To insure the consistency of this trial, the inclusion and exclusion criteria from another PCNSL trial (available on the Internet on www.ClinicalTrials.gov.) were adopted in this research as follows.

\section{Inclusion criteria}

(1) Histological confirmation of PCNSL, (2) Eastern Cooperative Oncology Group (ECOG) performance status of 0-1, (3) positive cerebrospinal fluid (CSF) cytology or immunohistochemical diagnosis of CSF monoclonality with or without measurable intracranial disease, (4) measurable
$(>1 \mathrm{~cm}$ in diameter) tumor by computed tomography (CT) scan or MRI, (5) progressed during first-line chemotherapy and/or radiotherapy OR relapsed after initial successful treatment, (6) no systemic lymphoma by CT scan of the chest, abdomen, and pelvis with contrast, (7) no leptomeningeal lymphoma by lumbar puncture for CNS cytology/flow cytometry, (8) no ocular lymphoma by slit-lamp examination, (9) must have adequate organ function as defined by the protocol: adequate renal function: serum creatinine $\leq 1.5 \mathrm{mg} / \mathrm{dL}$ and/or calculated creatinine clearance $\geq 60 \mathrm{~mL} / \mathrm{min}$; adequate hepatic function: bilirubin level $\leq 1.5 \times$ the upper limit of normal (ULN), aspartate aminotransferase (AST) and alanine aminotransferase $(\mathrm{ALT}) \leq 1.5 \times \mathrm{ULN}$. Adequate bone marrow reserves: neutrophil (ANC) count $\geq 1500 / \mathrm{mm}^{3}$, platelet count $\geq 100,000 / \mathrm{mm}^{3}$, and hemoglobin $\geq 9 \mathrm{~g} / \mathrm{dL}$. (10) Age $\geq 18$ and $\leq 75$ years. All patients signed written informed consent prior to study entry.

\section{Exclusion criteria}

(1) Patients with HIV seropositivity and systemic lymphoma manifestation, (2) serious uncontrolled concurrent illness, (3) previous brain radiotherapy and systemic chemotherapy, (4) concurrent chronic systemic immune therapy, targeted therapy not indicated in this study protocol, (5) any evidence of prior exposure to hepatitis B virus, (6) unable to comprehend the study requirements or who are not likely to comply with the study parameters, and (7) pregnant (confirmed by serum or urine $\beta$ human chorionic gonadotrophin $[\beta-\mathrm{HCG}]$ ) or lactating.

\section{Large doses of pemetrexed treatment regimen}

To improve safety and minimize pemetrexed-related toxicities, patients in our study were assigned to receive folic acid $0.4 \mathrm{mg}$ daily, beginning 1 week before the first dose of pemetrexed until 3 weeks after/treatment. In addition, patients also received dexamethasone $4 \mathrm{mg}$ orally twice a day beginning 1 day before until 1 day after treatment to prevent pemetrexed-induced rashes. Injections of $1 \mathrm{mg}$ vitamin $\mathrm{B}_{12}$ were also given intramuscularly 1-2 weeks before the first dose of pemetrexed and at 9-week intervals during the treatment. Patients were treated with pemetrexed at a dose of $900 \mathrm{mg} / \mathrm{m}^{2}$ intravenously every 3 weeks of a 6-week cycle. Patients remained on treatment as long as there was no evidence of tumor progression or intolerable side effects, and a complete response was not achieved. Moreover, 20\% of mannitol was injected intravenously into all patients at each time before and after the chemotherapy with a high rate for raising the permeability of blood-brain barrier. 


\section{The evaluation of response effects and side effects}

The baseline evaluation standard and response criteria for PCNSL formulated by the international lymphoma working group in 2005 were referenced as the response criteria in our study ${ }^{10}$ which included four levels for the response such as complete remission, partial remission, stable disease, and progressive disease. The overall response rate was calculated by the sum of complete remission and partial remission; however, the disease control rate was added by complete remission, partial remission, and stable disease rates. In addition, the side effects were assessed into $0-\mathrm{V}$ grades according to National Cancer Institute Common Terminology Criteria for Adverse Events (version 4.0), and toxicity was graded as mild (Grade 1), moderate (Grade 2), severe (Grade 3), life-threatening (Grade 4), or death (Grade 5).

\section{Follow-up}

Clinical follow-up and telephone interview were conducted with the patients until the end of April 31, 2015. MRI and MRS were performed in all patients. During the study period, the assessment of MRI and MRS was done every 3 months of the treatment, and patients would receive imaging examination immediately in case of severe symptoms.

\section{Statistical analysis}

The Kaplan-Meier method was adopted to calculate the survival curves, and all the statistical analysis were performed through SPSS 16.0.

\section{Results}

\section{Patient characteristics}

A total of 17 cases of recurrent PCNSL patients were enrolled in our study, including 10 males and 7 females with a median age of 66.2 years (ranging from 35 to 81 ) during the study period. The symptoms of the intracranial hypertension were observed in all 17 PCNSL recurrent patients, which included episodic headache (10 cases), emesis ( 5 cases), and blurred vision ( 2 cases). In addition, nine cases were observed with focal symptoms, such as limb movement disorder in five cases, visual disturbance in two cases, epileptic seizure in one case, and one case involved in paralysis or weakness in both legs, and sphincter disturbance due to spinal meninges metastases with cauda equine infiltration. The median score of Karnofsky performance status was 80 , which ranged from 60 to 90 (Table 1). All the patients had diffuse large B-cell lymphoma.

\section{Image features}

$\mathrm{T}_{1}$-weighted and $\mathrm{T}_{2}$-weighted images were performed for all the recurrent PCNSL patients. Figure 1 shows the representative MRI images for complete response patients before and after pemetrexed treatment. Moreover, the patients were examined every 3 months during the treatment.

\section{Outcome}

Among the 17 cases of recurrent PCNSL patients, 8 cases were dead during the tumor progression, including 5 cases died of the recurrent PCNSL and 3 cases died of infection.

Table I Patients clinical and treatment data

\begin{tabular}{|c|c|c|c|c|c|c|c|c|c|}
\hline Number & Age/sex & Position & KPS & NCC & IT & Response & Outcome & PFS & OS \\
\hline I & $72 / \mathrm{F}$ & PRI & 80 & 9 & $\mathrm{RT}+\mathrm{MTX}$ & CR & Alive & $15.4+$ & I5.4+ \\
\hline 2 & $49 / \mathrm{F}$ & ER & 80 & 8 & MTX & CR & Alive & $12.4+$ & $12.4+$ \\
\hline 3 & $67 / M$ & PRI & 80 & 9 & MTX & $C R$ & Alive & 10.1 & II.I+ \\
\hline 4 & $67 / F$ & ER & 80 & 7 & MTX & CR & Alive & $6.8+$ & $6.8+$ \\
\hline 5 & $67 / F$ & ER & 80 & 6 & $\mathrm{RT}+\mathrm{MTX}$ & CR & Alive & 6.2 & $7.5+$ \\
\hline 6 & $77 / M$ & ER & 80 & 10 & MTX & PR2 & Death & 11.2 & 11.2 \\
\hline 7 & $60 / F$ & ER & 80 & 7 & MTX & PR2 & Alive & $10.2+$ & $10.2+$ \\
\hline 8 & $76 / M$ & PRI & 70 & 8 & MTX & PR2 & Alive & 9.8 & 10.3 \\
\hline 9 & $45 / F$ & PRI & 90 & 2 & MTX & PR2 & Death & 7.8 & 8.6 \\
\hline 10 & $80 / F$ & PRI & 70 & 5 & MTX & PR2 & Alive & 6.2 & 7.8 \\
\hline II & $8 I / F$ & ER & 90 & 5 & $\mathrm{RT}$ & SD & Death & 3.8 & 7.1 \\
\hline 12 & $70 / M$ & ER & 60 & 4 & MTX & SD & Alive & 5.2 & 5.2 \\
\hline 13 & $69 / M$ & PRI & 70 & 3 & $\mathrm{RT}+\mathrm{MTX}$ & SD & Death & 1.8 & 4.2 \\
\hline 14 & $75 / M$ & ER & 80 & 3 & MTX & SD & Death & 2.1 & 3.9 \\
\hline 15 & $35 / M$ & MT & 70 & 3 & $\mathrm{RT}+\mathrm{MTX}$ & PD & Death & 6.6 & 6.6 \\
\hline 16 & $58 / F$ & ER & 70 & 4 & $R T+M T X$ & PD & Death & 5.7 & 5.7 \\
\hline 17 & $78 / M$ & PRI & 60 & 1 & $R T+M T X$ & PD & Death & 2.4 & 3.5 \\
\hline
\end{tabular}

Abbreviations: CR, complete remission; ER, ectopic recurrence; F, female; IT, initial treatment; KPS, Karnofsky performance status; M, male; MT, metastasis; MTX, methotrexate; NCC, number of chemotherapy cycles; OS, overall survival; PD, progressive disease; PFS, progression-free survival; PRI, primary recurrence; PR2, partial remission; $\mathrm{RT}$, radiotherapy; $\mathrm{SD}$, stable disease. 


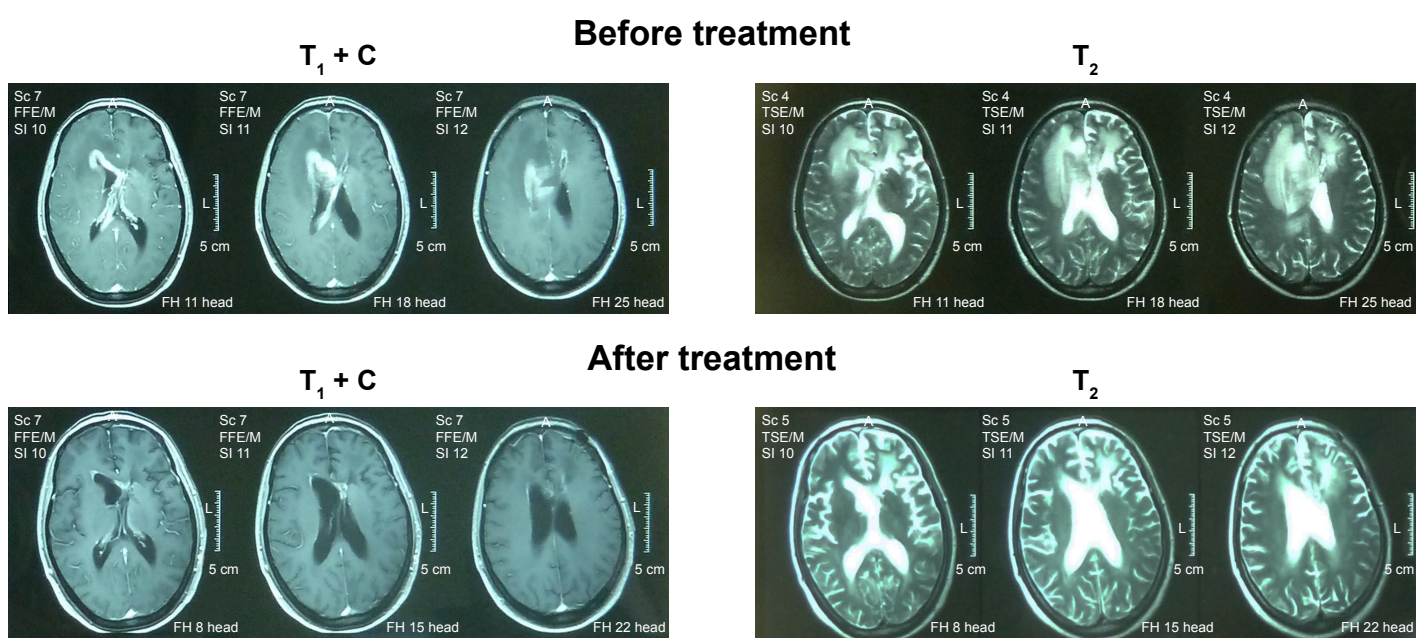

Figure I The representative magnetic resonance imaging images of complete response patient before and after the pemetrexed treatment.

After anti-infection treatment, the three patients with infection died of respiratory failure caused by drug-resistant bacteria pneumonia. Besides from the eight died cases, four cases were alive until the end of the study and five cases were alive until withdraw from the follow-up. All the patients received at least one cycle of chemotherapy with pemetrexed, and the average time of chemotherapy with pemetrexed was six cycles. After the treatment, five cases achieved complete remission, with partial remission in five cases, stable disease in four, and progressive disease in three cases. Consequently, the overall response rate was $58.8 \%$, and the disease control rate was $82.4 \%$. The median overall survival (OS) was 7.8 months $(95 \%$ confidence interval: 5.9-9.6 months) in 17 patients with recurrent PCNSL.

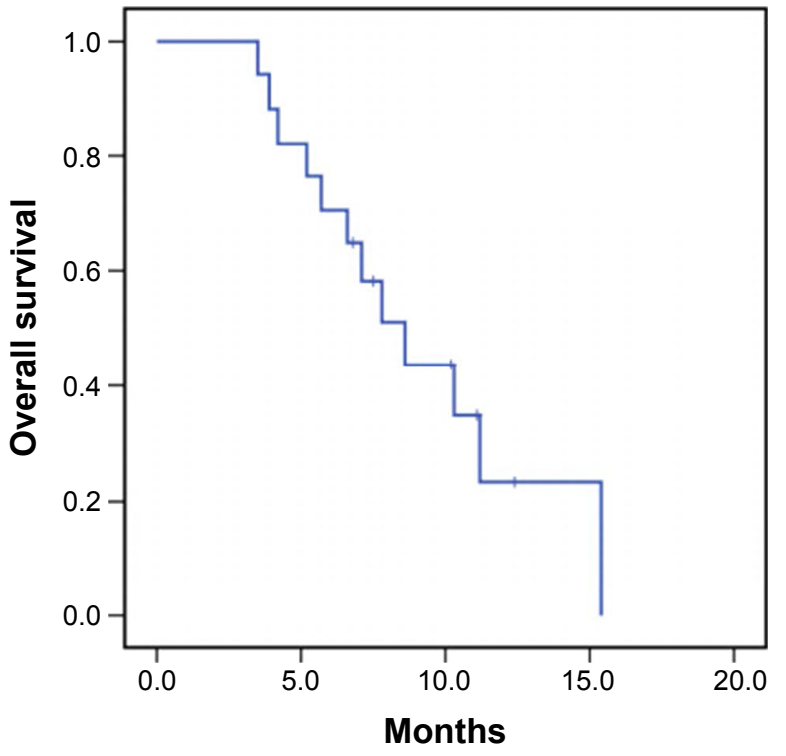

Figure 2 Overall survival of 17 recurrent primary central nervous system lymphoma patients treated with pemetrexed.
Moreover, Figure 2 shows the OS of 17 recurrent PCNSL patients treated with pemetrexed.

\section{Toxicities}

The adverse events mainly included myelosuppression, gastrointestinal reaction, and infection. After long-term chemotherapy, the patients had different degrees of myelosuppression symptoms presented as leukopenia, thrombocytopenia, and anemia, which included grades 1 and 2 in seven cases and grades 3 and 4 in three cases. The myelosuppression could be removed by drug withdrawal or symptomatic treatment. Nausea and vomiting, as the common gastrointestinal reaction, appeared in two (grades 2 and 3) and three (grades 1 and 3) cases, respectively. Moreover, nausea and vomiting could be remitted by receiving 5-hydroxytrptamin 3 antagonist. In addition, three patients died of infection, with other reactions including fatigue (five cases), constipation (four cases), and abnormal liver function (two cases). Specific adverse events were recorded in Table 2.

\section{Discussion}

PCNSL is a highly invasive and destructive malignant brain tumor with rapid disease progression. Due to the similar clinical presentation and radiological features compared with other CNS mass lesions, establishing the diagnosis of PCNSL is difficult as well as achieving the effective treatment with good prognosis. Doucet et al reported that after the confirmation of PCNSL diagnosis, there were 35\%-60\% patients recurrent 2 years later and the prognosis was poor in patients with median OS time for 8-18 months. ${ }^{11}$ Although many reports have described the use of RT or chemotherapy with varying degrees of activity, there has been still no 
Table 2 Adverse events associated with pemetrexed $(\mathrm{N}=17)$

\begin{tabular}{llllll}
\hline Toxicity & Grade I & Grade 2 & Grade 3 & Grade 4 & Grade 5 \\
\hline Leukoencephalopathy & 0 & 0 & 0 & 0 & 0 \\
ALT/AST & 0 & 1 & 1 & 0 & 0 \\
Infection & 0 & 0 & 0 & 0 & 3 \\
Anemia & 2 & 0 & 0 & 1 & 0 \\
Leukopenia & 0 & $\mathrm{I}$ & $\mathrm{I}$ & $\mathrm{I}$ & 0 \\
Thrombocytopenia & 3 & $\mathrm{I}$ & $\mathrm{I}$ & 0 & 0 \\
Nausea & 0 & $\mathrm{I}$ & 2 & 0 & 0 \\
Vomiting & $\mathrm{I}$ & 0 & $\mathrm{I}$ & 0 & 0 \\
Fatigue & 2 & 2 & $\mathrm{I}$ & 0 & 0 \\
Constipation & 2 & $\mathrm{I}$ & & & 0 \\
\hline
\end{tabular}

Abbreviations: ALT, alanine aminotransferase; AST, aspartate aminotransferase.

standard therapeutic regimens for PCNSL. In our study, this is the first report of using pemetrexed in recurrent PCNSL in China. Considering the significant need for effective salvage therapies in recurrent PCNSL patients, our study was aimed to assess the efficacy and safety of pemetrexed in these patients.

At present, there has been no universal consensus about the treatment of recurrent PCNSL. In recent years, many new medicines, such as temozolomide and rituximab, have been widely used in the recurrent PCNSL patients of multicenter clinical trials. ${ }^{12}$ Wong et $\mathrm{l}^{13}$ applied only temozolomide to treat the seven PCNSL recurrent patients, and the results showed that the progression-free survival time was 2 months, followed by 4 months for OS time with the effective rate of $14 \%$. Nayak et al ${ }^{14}$ carried out a phase II multicenter clinical trial combining temozolomide and rituximab to treat the recurrent PCNSL patients, and their study suggested that the complete remission rate was $14 \%$ and the central progressionfree survival rate was 7 weeks. However, results of research were not expectative.

Pemetrexed, an antitumor drug, has the advantage of targeting more than 1 sites in the folate metabolism compared with methotrexate. Moreover, it could interrupt purine synthesis via thymidylate synthase and dihydrofolate reductase inhibition, and pyrimidine synthesis via glycinamide ribonucleotide formyltransferase and aminoimidazole carboxamide formyltransferase inhibition without hydration, alkalization, and detoxication. ${ }^{15}$ Raizer et $\mathrm{al}^{8}$ performed a research to study the content of pemetrexed in the CSF of patients, and they found that the CSF content of pemetrexed was $1 \%-3 \%$ of plasma, which was low but could target the tumor cells and be efficiently tumoricidal. According to the advantages of pemetrexed, it was considered that pemetrexed may have a better efficacy for recurrent PCNSL treatment. To our knowledge, there have been only two studies involved in the efficacy of pemetrexed for recurrent
PCNSL patients around the world. A research program funded by U.S. National Cancer Institute and Northwestern University revealed that after five cycles of chemotherapy with $900 \mathrm{mg} / \mathrm{m}^{2}$ pemetrexed treatment for recurrent PCNSL patients, the 6-month progression-free survival rate, response rate, and disease control rate were $45 \%, 55 \%$, and $91 \%$, respectively. ${ }^{8}$ Zhang et al ${ }^{9}$ performed a retrospective study to review the outcomes and toxicities of pemetrexed with modified doses for recurrent PCNSL patients, which indicated that pemetrexed has activity in recurrent PCNSL with modest toxicity. In addition, the results of this study showed that the response rate was $64.7 \%$, and the OS was 22.6 months. In our study, results in the recurrent PCNSL patients were comparable with a response rate of $58.8 \%$, and the median OS of 7.8 months. According to the dose of pemetrexed with $900 \mathrm{mg} / \mathrm{m}^{2}$, the toxicity seen in our patients was higher than in prior studies, which may be a result of the high standard dose and degree of initial therapy with three infectious deaths. The increased toxicity may be related with the high dose, and it may be decreased through less standard dose.

Limitations of this study should be acknowledged. One of the limitations is that our study, a quasi-experiment, has inherent limitations including lack of randomization and control. In addition, the differences in patient populations existed in our study which may affect the results potentially.

\section{Conclusion}

This study has been the first clinical trial, which applied pemetrexed to treat recurrent PCNSL patients in China, and results indicated that the chemotherapy using large pemetrexed may become an effective treatment for PCNSL recurrence with modest toxicity. However, due to lack of enough cases and experience, it should be further confirmed through multicenter large-scale clinical trials. 


\section{Acknowledgments}

This work was supported by the Shandong Cancer Hospital, and we thank for sharing the data and funding needs for this study.

\section{Disclosure}

The authors report no conflicts of interest in this work.

\section{References}

1. Korfel A, Schlegel U. Diagnosis and treatment of primary CNS lymphoma. Nat Rev Neurol. 2013;9(6):317-327.

2. Shen L, Chen BB, Chen Z, Xv XP, Lin GW. Expression of FOXP1 and Cydin $\mathrm{E}$ in primary central nervous system lymphoma and its significance. Chin J Hematol. 2012;33(8):648-652. Chinese.

3. Hottinger AF, DeAngelis LM, Yahalom J, Abrey LE. Salvage whole brain radiotherapy for recurrent or refractory primary CNS lymphoma. Neurology. 2007;69(11):1178-1182.

4. Voloschin AD, Betensky R, Wen PY, Hochbery F, Batchelor T. Topotecan as salvage therapy for relapsed or refractory primary central nervous system lymphoma. J Neurooncol. 2008;86(2):211-215.

5. Enting RH, Demopoulos A, DeAngelis LM, Abrey LE. Salvage therapy for primary CNS lymphoma with combination of rituximab and temozolomide. Neurology. 2004;63(5):901-903.

6. Reni M, Zaja F, Mason W, et al. Temozolomide as salvage treatment in primary brain lymphoma. Br J Cancer. 2007;96(6):864-867.
7. Plotkin SR, Betensky RA, Hochberg FH, et al. Treatment of relapsed central nervous system lymphoma with high-dose methotrexate. Clin Cancer Res. 2004;10(17):5643-5646.

8. Raizer JJ, Rademaker A, Evens AM, et al. Pemetrexed in the treatment of relapsed/refractory primary central nervous system lymphoma. Cancer. 2012;118(15):3743-3748.

9. Zhang JP, Lee EQ, Nayak L, et al. Retrospective study of pemetrexed as salvage therapy for central nervous system lymphoma. $J \mathrm{Neu}$ rooncol. 2013;115(1):71-77.

10. Abrey LE, Batchelor TT, Ferreri AJ, et al; International Primary CNS Lymphoma Collaborative Group. Report of an international workshop to standardize baseline evaluation and response criteria for primary CNS lymphoma. J Clin Oncol. 2005;23(22):5034-5043.

11. Doucet S, Kumthekar P, Raizer J. Primary central nervous system lymphoma. Curr Treat Options Oncol. 2013;14:185-187.

12. Birnbaum T, Stadler EA, Baumgarten L, Straube A. Rituximab significantly improves complete response rate in patients with primary CNS lymphoma. J Neurooncol. 2012;109(2):285-291.

13. Wong SF, Gan HK, Cher L. A single center study of the treatment of relapsed primary central nervous system lymphoma (PCNSL) with single agent temozolomide. J Chin Neurosci. 2012;19(11):1501-1505.

14. Nayak L, Abrey LE, Drappatz J, et al. Multicenter phase II study of rituximab and temozolomide in recurrent primary central nervous system lymphoma. Leuk Lymphoma. 2013;54(1):58-61.

15. Argiris A, Pennella E, Koustenis A, Hossain AM, Obasaju CK. Pemetrexed in head and neck cancer: a systematic review. Oral Oncol. 2013;49(6):492-501.
OncoTargets and Therapy

\section{Publish your work in this journal}

OncoTargets and Therapy is an international, peer-reviewed, open access journal focusing on the pathological basis of all cancers, potential targets for therapy and treatment protocols employed to improve the management of cancer patients. The journal also focuses on the impact of management programs and new therapeutic agents and protocols on

\section{Dovepress}

patient perspectives such as quality of life, adherence and satisfaction. The manuscript management system is completely online and includes a very quick and fair peer-review system, which is all easy to use. Visit http://www.dovepress.com/testimonials.php to read real quotes from published authors. 